\title{
Estudio Descriptivo de la Irrigación de la Tuberosidad Tibial y Cresta Tibial en Muestras Cadavéricas de Perros (Canis lupus familiaris), Mediante Repleción Arterial con Tinta China y Medio de Contraste Radiográfico
}

\author{
Descriptive Study of Tibial Tuberosity and Tibial Crest Blood Supply in Dog Carcass Samples \\ (Canis lupus familiaris) by Arterial Injection with Indian Ink and Radiographic Contrast
}

Oliver Quintana-García ${ }^{1}$; Ismael Concha-Albornoz²; Pablo Fariña-Sirandoni² \& Molly Addison ${ }^{2}$

QUINTANA-GARCÍA, O.; CONCHA-ALBORNOZ, I.; FARIÑA-SIRANDONI, P. \& ADDISON, M. Estudio descriptivo de la irrigación de la tuberosidad tibial y cresta tibial en muestras cadavéricas de perros (Canis lupus familiaris), mediante repleción arterial con tinta china y medio de contraste radiográfico. Int. J. Morphol., 36(2):407-412, 2018.

RESUMEN: La ruptura del ligamento cruzado craneal corresponde a la causa más común de cojera en el canino, siendo poco clara su etiología. En la práctica el diagnóstico de la patología se realiza mediante pruebas clínicas ortopédicas, las que corresponden a la prueba de cajón y la prueba de compresión tibial. El desarrollo de diferentes soluciones quirúrgicas incorpora osteotomías a nivel de la tuberosidad tibial, proceso que presenta riesgos frente a una isquemia ósea postquirúrgica. Las descripciones de la anatomía vascular de esta zona de la articulación son escasas y permanecen pobremente representadas en los textos de anatomía. El objetivo de este estudio fue describir el origen de la irrigación de la epífisis proximal y diáfisis en su tercio proximal de la tibia en el perro, mediante la inyección de medio de contraste mezclado con tinta china a través de dos vías arteriales diferentes, incluyendo una radiografía simple previa a la inyección y otra contrastada posterior a la inyección. Se evaluó un total de 48 miembros pélvicos de perros, organizados en tres grupos. En todos los grupos se obtuvo presencia de tinción en la tuberosidad tibial, tanto por vasos originados desde la arteria poplítea, así como también por vasos originados desde la arteria femoral.

PALABRAS CLAVE: Anatomía; Perro; Irrigación; Tibia.

\section{INTRODUCCIÓN}

La rodilla corresponde a una articulación sinovial compleja del subtipo gínglimo, que puede realizar los movimientos de flexo - extensión y limitado desplazamiento medio - lateral (König \& Liebich, 2005), ubicada en el miembro pélvico. Funcionalmente corresponde a una articulación en donde los diagnósticos de patologías son frecuentemente de mayor complejidad. La articulación incluye dos ligamentos denominados "cruzados", debido a su disposición en forma de X, donde elligamento cruzado craneal posee 2 bandas, una antero-medial más gruesa y retorcida, y una posterolateral más delgada y recta (Córdoba et al., 2007; Álvarez, 2011), ambos mantienen la estabilidad articular, impidiendo la rotación medial, desplazamiento craneal de la tibia y la hiperextensión de la rodilla (Álvarez). La ruptura de ligamento cruzado craneal corresponde a la causa más común de cojera en el canino (Steinberg et al., 2011). Su diagnósti- co incorpora pruebas clínicas llamadas "prueba de cajón" y "prueba de compresión tibial", las que son positivas durante el examen clínico del paciente con la patología, y que pueden ser apoyadas por radiografía (simple y bajo compresión tibial), ecografía, artroscopía y resonancia nuclear magnética.

La causa definitiva de la patología aun no es clara, pero se manejan ciertos factores como la edad, la genética, obesidad, e incluso mecanismos inmunes como elementos vinculados a la enfermedad. Los tratamientos de la enfermedad incluyen el uso de medicamentos y reposo del paciente (terapia conservadora), y otros más actuales realizan tratamientos quirúrgicos. En estos últimos existen dos líneas de tratamiento quirúrgico: simular la función del ligamento cruzado craneal, mientras que la otra modifica la

\footnotetext{
${ }^{1}$ Escuela de Medicina Veterinaria, Universidad Santo Tomás (UST), Santiago de Chile, Chile.

${ }^{2}$ College of Veterinary Medicine, Lincoln Memorial University (LMU), Tennessee, Estados Unidos de América.
} 
QUINTANA-GARCÍA, O.; CONCHA-ALBORNOZ, I.; FARIÑA-SIRANDONI, P. \& ADDISON, M. Estudio descriptivo de la irrigación de la tuberosidad tibial y cresta tibial en muestras cadavéricas de perros (Canis lupus familiaris), mediante repleción arterial con tinta china y medio de contraste radiográfico. Int. J. Morphol., 36(2):407-412, 2018.

biomecánica de la articulación (Rodríguez Quirós et al., 2008). Si bien existen terapias para tratar la enfermedad, sus efectos confluyen a un mismo camino, la osteoartritis de la articulación afectada (Cook, 2010).

Un ejemplo de las técnicas modernas que modifican la biomecánica de la articulación es llamada "Avance de la Tuberosidad Tibial" (TTA de sus siglas en inglés "Tibial Tuberosity Advancement”) y la “Osteotomía niveladora de Platillo Tibial (TPLO de sus siglas en inglés "Tibial Plateau Leveling Osteotomy").

El éxito de las cirugías, en general, depende de una serie de factores. Uno de ellos es la comprensión de la anatomía y fisiología de la especie a tratar. La necrosis del fragmento de hueso tibial producto de la osteotomía quirúrgica se ha descrito y es motivo para estudiar la irrigación de la tuberosidad tibial en el canino, y pese a que existen trabajos en donde se detalla un mapeo de la arquitectura vascular del miembro pélvico, aun no se dilucida en forma clara la irrigación del hueso tibial en su porción más proximal, ni tampoco existe información clara en los textos de anatomía (Kanamiya et al., 2001).

La irrigación de la región de la rodilla está dada por la arteria femoral, rama directa de la arteria iliaca externa, así como también por la arteria poplítea, la cual es la continuación de la arteria femoral hacia distal de la articulación genual. La arteria poplítea se continúa hacia distal para originar dos ramas: la arteria tibial craneal, que se lateraliza, y se dispone entre la tibia y fíbula hacia craneal e irriga los músculos fibular largo, extensor digital común y tibial craneal; y la arteria tibial caudal, vaso pequeño que nace de la superficie caudal de la art. poplítea en el espacio interóseo (Evans \& Delahunta, 2002).

Además, se describe la arteria nutricia tibial, con origen en la arteria tibial caudal que, después de penetrar el hueso, se divide en dos ramas medulares, que son los principales suministros de sangre para la tibia (Dehghani et al., 2004). La arteria nutricia tibial y sus ramas ascendentes y descendentes fueron observadas claramente en un estudio después de cinco segundos de inyectado el medio de contraste, estas ramas originaron ramas más finas que recorrieron longitudinalmente hasta alcanzar la región metafisiaria de la tibia (Kato et al., 1970). Estudios en medicina humana señalan el uso de la radiografía contrastada como una herramienta diagnóstica para enfermedades como el atrapamiento de la arteria poplítea (Parra et al., 2006) y enfermedades arteriales causantes de isquemia del miembro inferior (AhChong et al., 1999).

Este estudio tuvo por objetivo describir el origen de la irrigación de la epífisis proximal así como también el tercio proximal de la diáfisis de la tibia en cadáveres de perro. Nosotros planteamos que la irrigación de la tibia proviene de la arteria nutricia tibial y que puede existir aporte sanguíneo desde tejidos blandos adyacentes a ella. Para esto se realizaron inyecciones de tinta china mezclada con medio de contraste a través de vasos arteriales principales y constantes en el canino, para así conocer su aporte en la irrigación de la tibia mediante el uso de radiografía contrastada y la observación mesoscópica de la tinta en cortes de los miembros pélvicos.

Nuestra hipótesis es que la irrigación a través de tejidos blandos que recibe la tuberosidad tibial desde ramas de origen femoral proximal a la arteria poplítea, sería suficiente para nutrir dicha parte del hueso posterior a su osteotomía.

Los resultados sugieren que la separación de la tuberosidad tibial del resto de la tibia, mediante osteotomía quirúrgica, no afectaría la irrigación y vitalidad de la misma. Además, se observó la existencia de permeabilidad de vasos sanguíneos desde tejido blando adyacente hacia el hueso esponjoso de la tuberosidad tibial.

\section{MATERIAL Y MÉTODO}

Se utilizaron 48 miembros pélvicos de canino adulto, con peso corporal entre los 20 a $45 \mathrm{Kg}$, de cualquier sexo y raza, libres de trastornos ortopédicos, sin alteraciones clínicas visibles y/o radiológicas en la articulación de la rodilla. Los 48 miembros pélvicos de canino fueron organizados en 3 grupos de 16 miembros pélvicos cada uno. Posteriormente se realizó un abordaje de las vías arteriales y un lavado previo de las vías vasculares con agua tibia para luego proceder a la inyección de tinta china mezclada con medio de contraste Omnipaque ${ }^{\circledR} 300 \mathrm{mg} \mathrm{1/ml} \mathrm{(100} \mathrm{ml).}$

Para cada grupo se utilizó la siguiente metodología:

Grupo 1: Inyección de 4 cc de tinta china color azul mezclado con 2 cc de medio de contraste a través de la arteria femoral y ligadura de la art. poplítea.

Grupo 2: Inyección de 4 cc de tinta china color amarillo mezclado con 2 cc de medio de contraste vía arteria poplítea y ligadura proximal al punto de canulación.

Grupo 3: Inyección vía arteria femoral y poplítea con 4 cc de tinta china color azul y amarillo respectivamente, ambas mezcladas con $2 \mathrm{cc}$ de medio de contraste, manteniendo la ligadura proximal al punto de canulación (como en el grupo 2), siendo inyectadas de manera simultánea para buscar po- 
sibles áreas de irrigación en común mediante la mezcla de éstas originando una coloración verde.

Se utilizó cautín eléctrico tipo lápiz 80 watts Macrotel ${ }^{\circledR}$ para cauterizar vasos sanguíneos de tejidos blandos seccionados al realizar el abordaje de la arteria poplítea para evitar el escurrimiento de producto inyectado.

Abordaje de la arteria femoral: Se realizó considerando como referencia el trígono femoral, el cual está limitado en su parte craneal por el $\mathrm{m}$. sartorio, caudalmente por el $\mathrm{m}$. pectíneo y en profundidad por el $\mathrm{m}$. iliopsoas y el $\mathrm{m}$. vasto medial (Evans \& Delahunta). Para acceder al trígono femoral se realizó una incisión en el tegumento de la región femoral paralelo al músculo sartorio. Se expuso tejido subcutáneo y adiposo de la región hasta ubicar la arteria femoral.

Abordaje de la arteria poplítea: Se realizó una incisión en la superficie medial de la región genual, inicialmente paralela al fémur, para luego ser curva y paralela a la tibia a nivel del margen caudal de la porción craneal del músculo sartorio, cercano a la articulación de la rodilla. Se disecó tejido subcutáneo y adiposo para exponer el músculo sartorio, el que se rechazó hacia caudal, posteriormente se seccionó el músculo semimembranoso en su inserción en el cóndilo medial del fémur y se rechazó hacia proximal. Finalmente se abordó la fosa poplítea, canulando la a. poplítea con mariposa $21 \mathrm{G}$ para la inyección de la mezcla o para su ligadura, según el grupo a trabajar. Una vez inyectados los miembros se realizaron radiografías (contrastadas) para visualizar el recorrido del producto inyectado.

Radiografías: Fueron realizadas con el equipo Siemens ${ }^{\circledR}$ Nanodor 2, incorporando las vistas cráneo-caudal y la medio-lateral. Los valores de kilovoltaje $(\mathrm{kV})$ y tiempo (s) para cada vista se calcularon según la siguiente fórmula (Mendoza, 2001):

- Vista cráneo-caudal: $\mathrm{kV}=($ diámetro del miembro x 2)+40 y 0,10 s

- Vista medio-lateral: $\mathrm{kV}=($ diámetro del miembro x 2)+40 y $0.12 \mathrm{~s}$

Los valores se mantuvieron para las radiografías simples y contrastadas. Posteriormente los miembros inyectados fueron congelados $\left(-20^{\circ} \mathrm{C}\right)$ para ser cortados mediante sierra circular en direcciones sagital, transversal y frontal, fijados en formalina al $10 \%$ y evaluar los territorios irrigados por cada arteria mediante la visualización mesoscópica del recorrido de la tinta china con la ayuda de lupa con fuente lumínica. De los 48 miembros en estudio, 30 fueron trabajados en la Unidad de Anatomía Veterinaria de la Universidad Santo Tomás Sede Santiago (Chile) y 18 en el Labora- torio de Anatomía Veterinaria de Lincoln Memorial University (Tennessee, EE.UU.). En cada uno de los 3 grupos se realizaron 10 miembros con cortes transversales, 3 miembros con cortes sagitales y 3 con cortes frontales.

\section{RESULTADOS}

Grupo 1. Se observó presencia de tinta china azul en el tejido esponjoso de la tuberosidad tibial en todas las muestras, así como también la presencia de ramas medulares, bien delimitadas en cortes sagitales y regiones con tinción difusa (Fig. 1). Una pieza presentó hasta 5 ramas medulares. Los vasos arteriales observados en la radiografía contrastada fueron la arteria femoral, a. safena, a. femoral caudal media y a. femoral caudal distal originando sus ramas hacia caudal. No se observó la arteria nutricia tibial en las muestras. Cinco muestras presentaron manchas difusas de medio de contraste extravasado ("nube") en las zonas donde se realizó la ligadura de la arteria poplítea.

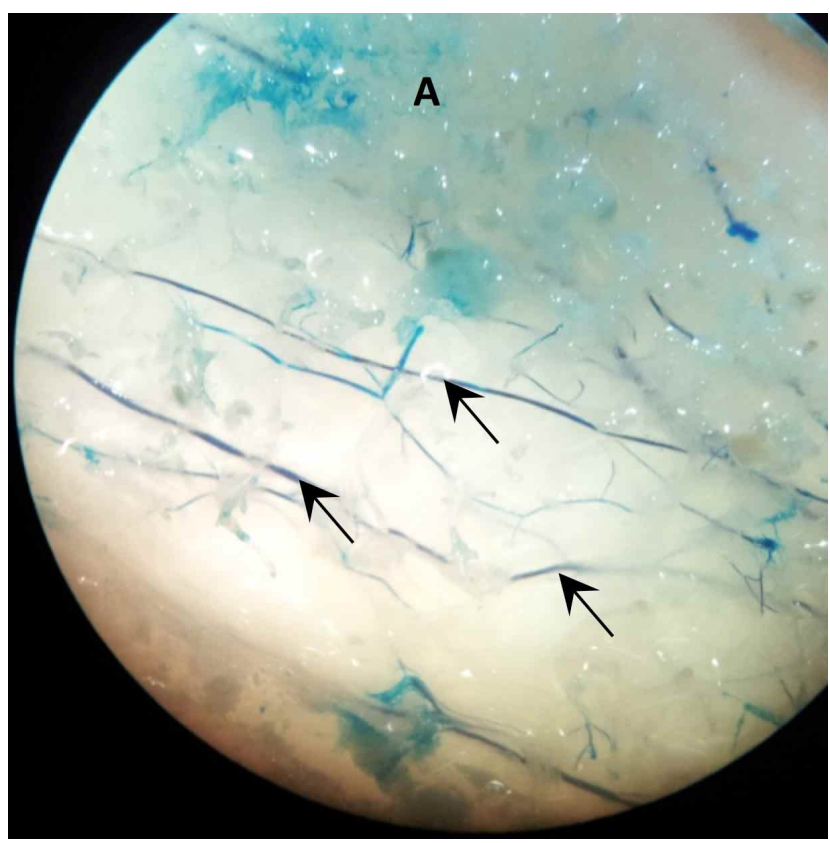

Fig. 1. Corte frontal en tuberosidad tibial. Las flechas señalan ramas medulares pequeñas; A: región de tinción difusa.

Grupo 2. Se observó presencia de tinción de color amarillo en el tejido esponjoso de la tuberosidad tibial de todas las muestras, excepto en una (cadáver 20, miembro pélvico derecho). Se observaron hasta 4 ramas medulares y la a. nutricia tibial en 2 muestras (Fig. 2). En todas las radiografías se observó claramente las arterias poplítea y tibial craneal teñidas con medio de contraste. En una sola muestra se 
visualizó la a. nutricia tibial entrando al canal medular tibial a través del agujero nutricio tibial (Fig. 4).

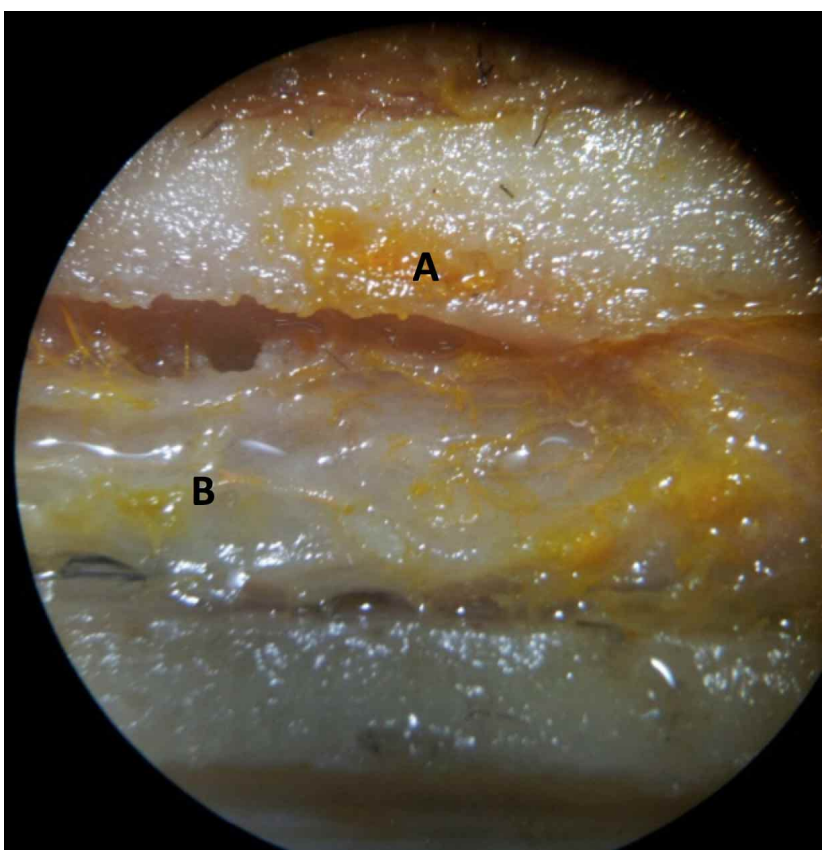

Fig. 2. Corte frontal en tercio proximal de diáfisis. A: canal nutricio por donde penetra la arteria nutricia tibial hacia la tibia. B: ramas medulares de menor calibre.

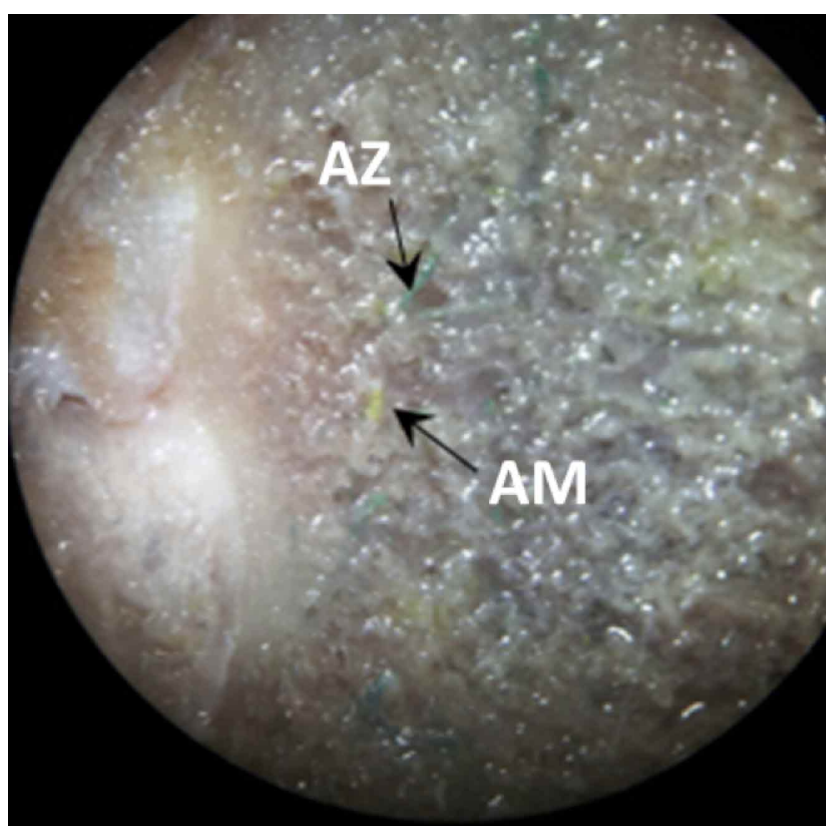

Fig. 3. Corte sagital de tuberosidad tibial, tibia izquierda (muestra 21). Las flechas señalan vasos teñidos de color azul (AZ) y amarillo (AM).

Grupo 3. En dos muestras fue posible observar 5 ramas arteriales en tejido esponjoso de la tuberosidad tibial (Tabla I). En 5 muestras se observaron vasos de ambos colores, de

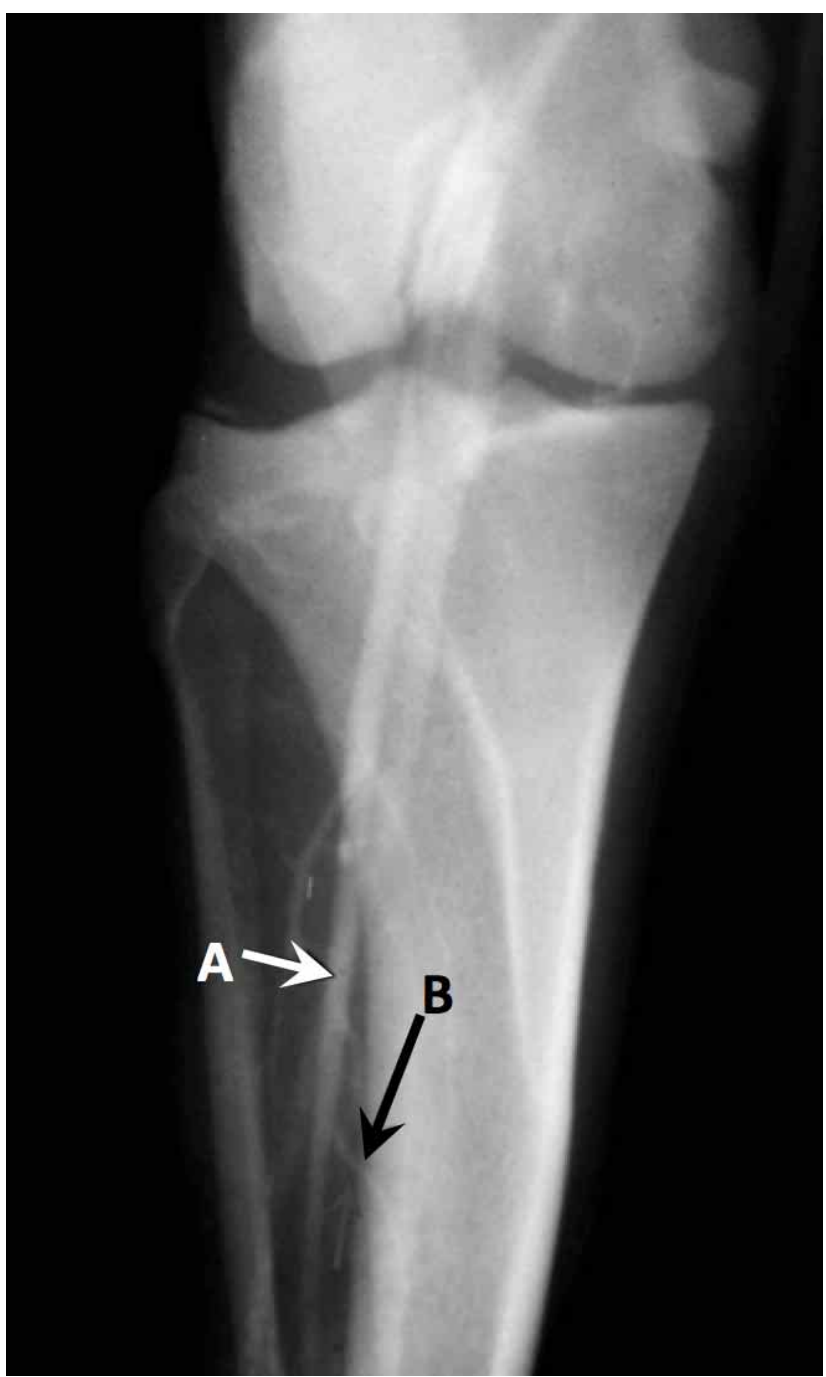

Fig. 4. Arteriografía vía arteria poplítea (muestra 11, UST), vista cráneo-caudal de articulación genual. A: arteria tibial caudal. B: art. nutricia tibial entrando a través del agujero nutricio tibial.

forma independiente, pero siempre con predominio de ramas arteriales teñidas de amarillo (Fig. 3). Los vasos de color azul siempre se observaron a nivel proximal en la tuberosidad tibial, mientras que vasos con color amarillo fueron observados hacia distal.

\section{DISCUSIÓN}

La presencia de dos o más ramas medulares difiere de lo expresado en los estudios de Dehghani et al. quien señala la existencia de dos únicas ramas arteriales medulares. El estudio radiográfico ratificó el recorrido de la art. poplítea y las ramas que de ella emergen hacia la rodilla y musculatura caudal de la tibia proximal según lo descrito por Evans 
\& Delahunta. La visualización del grupo de arterias geniculares concuerda con Kanamiya et al., dejando en evidencia que el suministro sanguíneo de la rodilla proviene de la anastomosis de las mismas.

Podemos mencionar que la precisión de nuestros estudios radiográficos podría ser mejorada con un cálculo más preciso de la cantidad, concentración y dilución de medio de contraste utilizado en cada muestra mezclado con tinta china, lo que creemos pudo tener influencia sobre la calidad del estudio. La técnica radiográfica no cumplió con nuestras expectativas, debido a su baja precisión, metodología que puede ser reemplazada por herramientas de mayor precisión, como por ejemplo el uso de tomografía axial computarizada (TAC). La mezcla con tinta china aumenta la dilución del medio de contraste, lo que va en desmedro de la técnica radiográfica, resultando imágenes poco claras para su análisis.

Nuestra experiencia con la radiografía contrastada difiere con la de AhChong et al. y Parra et al., quienes exponen casos clínicos en donde la técnica demuestra la presencia de anormalidad y enfermedad vascular, y se correlacionan con lo visto durante las cirugías en determinados casos del estudio.

La visualización de ramas medulares observadas en radiografías contrastadas de los tres grupos estudiados ratifica lo expresado por Kato et al., quien describió la presencia de ramas medulares más finas que se distribuyen de forma longitudinal hasta llegar a la región metafisiaria de la tibia y que serían originadas de la arteria nutricia tibial. En congruencia con nuestros resultados, la arteria nutricia tibial fue visible en las radiografías contrastadas, mientras que las ramas medulares fueron visibles emergiendo de esta para alcanzar e ingresar a la tibia, lo que evidencia la existencia de vasos sanguíneos que proporcionan la irrigación hacia la tuberosidad tibial.

En los estudios de Kato et al., AhChong et al. y Parra et al. los pacientes, tanto cánidos como humanos, permanecían vivos, lo que facilitó la circulación del medio de contraste a través del sistema circulatorio, además de ir en forma pura, sin la adición de tinta china, lo que puede explicar los resultados satisfactorios de la técnica de radiográfica para los estudios antes mencionados.

\section{CONCLUSIONES}

El tercio proximal de la diáfisis tibial y la epífisis proximal de la misma se mantienen irrigadas a través de un suministro sanguíneo proveniente de ramas originadas por la a. femoral, que llegan a través de tejidos blandos adyacentes a la epífisis proximal de la tibia. La a. nutricia tibial predomina en la irrigación de la tibia en función de la cantidad de vasos originados en el canal medular, y por ende, es el principal aporte sanguíneo a la tibia en el tercio proximal de la diáfisis, mientras que el aporte sanguíneo que llega hacia la epífisis proximal de la misma proviene desde tejido blando adyacente a la tibia.

Tabla I. Cuadro resumen de las ramas medulares observadas en forma mesoscópica en las tuberosidades tibiales de cada grupo.

\begin{tabular}{|c|c|c|c|c|c|c|c|c|c|}
\hline & \multicolumn{3}{|c|}{$\begin{array}{c}\text { Grupo } 1 \\
\text { Inyección vía A. femoral }\end{array}$} & \multicolumn{3}{|c|}{$\begin{array}{c}\text { Grupo } 2 \\
\text { Inyección vía A. poplítea }\end{array}$} & \multicolumn{3}{|c|}{$\begin{array}{c}\text { Grupo } 3 \\
\text { Invección vía } \mathrm{A} \text {. }\end{array}$} \\
\hline & Miembros & Corte & $\mathrm{N}^{\mathrm{o}}$ de Ramas & Miembros & Corte & $\mathrm{N}^{\circ}$ de Ramas & Miembros & Corte & $\mathrm{N}^{\circ}$ de Ramas \\
\hline \multirow{9}{*}{$\sqrt{\sigma}$} & $\mathrm{D}$ & $\mathrm{T}$ & 1 & $\mathrm{D}$ & $\mathrm{T}$ & 2 & I & $\mathrm{T}$ & 4 \\
\hline & $\mathrm{D}$ & $\mathrm{T}$ & 5 & I & $\mathrm{T}$ & 2 & I & $\mathrm{T}$ & 2 \\
\hline & I & $\mathrm{T}$ & 1 & $\mathrm{D}$ & $\mathrm{T}$ & Difuso & I & $\mathrm{T}$ & 3 \\
\hline & I & $\mathrm{T}$ & Difuso & I & $\mathrm{T}$ & Difuso & $\mathrm{D}$ & $\mathrm{T}$ & 2 \\
\hline & $\mathrm{D}$ & $\mathrm{S}$ & Difuso & $\mathrm{D}$ & $\mathrm{F}$ & 2 & I & $\mathrm{S}$ & 3 \\
\hline & $\mathrm{D}$ & $\mathrm{S}$ & 2 & I & $\mathrm{F}$ & 4 & $\mathrm{D}$ & $\mathrm{S}$ & 5 \\
\hline & I & $\mathrm{F}$ & 4 & $\mathrm{D}$ & $\mathrm{F}$ & 1 & $\mathrm{D}$ & $\mathrm{F}$ & 3 \\
\hline & $\mathrm{D}$ & $\mathrm{S}$ & 1 & I & S & 2 & I & $\mathrm{F}$ & 0 \\
\hline & I & $\mathrm{F}$ & 1 & $\mathrm{D}$ & S & 4 & I & $\mathrm{S}$ & 5 \\
\hline \multirow{7}{*}{$\underset{⿱ ㇒}{\sum_{\exists}}$} & $\mathrm{D}$ & $\mathrm{F}$ & Difuso & I & S & 2 & $\mathrm{D}$ & $\mathrm{F}$ & 2 \\
\hline & $\mathrm{I}$ & $\mathrm{S}$ & Difuso & I & $\mathrm{T}$ & 4 & $\mathrm{D}$ & $\mathrm{T}$ & Difuso \\
\hline & $\mathrm{D}$ & $\mathrm{T}$ & 2 & I & $\mathrm{T}$ & Difuso & $\mathrm{D}$ & $\mathrm{T}$ & Difuso \\
\hline & I & $\mathrm{T}$ & 4 & $\mathrm{D}$ & $\mathrm{T}$ & 0 & I & $\mathrm{T}$ & 1 \\
\hline & $\mathrm{D}$ & $\mathrm{T}$ & 1 & I & $\mathrm{T}$ & Difuso & I & $\mathrm{T}$ & Difuso \\
\hline & $\mathrm{D}$ & $\mathrm{T}$ & 2 & I & $\mathrm{T}$ & 1 & $\mathrm{D}$ & $\mathrm{T}$ & 1 \\
\hline & $\mathrm{D}$ & $\mathrm{T}$ & Difuso & I & $\mathrm{T}$ & Difuso & I & $\mathrm{T}$ & 1 \\
\hline
\end{tabular}

Donde D: derecho, I: izquierdo, T: transversal, S: sagital, F: frontal. 
QUINTANA-GARCÍA, O.; CONCHA-ALBORNOZ, I.; FARIÑA-SIRANDONI, P. \& ADDISON, M. Estudio descriptivo de la irrigación de la tuberosidad tibial y cresta tibial en muestras cadavéricas de perros (Canis lupus familiaris), mediante repleción arterial con tinta china y medio de contraste radiográfico. Int. J. Morphol., 36(2):407-412, 2018.

En el presente estudio no se logró evidenciar anastomosis entre las ramas arteriales provenientes de la arteria femoral con las provenientes de la arteria poplítea a nivel de la tuberosidad tibial. La presencia de tinta china de color azul inyectada vía art. femoral con ligadura proximal a la art. poplítea, es clara demostración de la existencia de vasos sanguíneos permeables desde el tejido blando adyacente (especialmente desde la musculatura) hacia tejido esponjoso de la tuberosidad tibial.

Los resultados obtenidos sugieren que la separación de la tuberosidad tibial del resto de la tibia, mediante una osteotomía, no afectaría su irrigación y vitalidad.

QUINTANA-GARCÍA, O.; CONCHA-ALBORNOZ, I.; FARIÑA-SIRANDONI, P. \& ADDISON, M. Descriptive study of tibial tuberosity and tibial crest blood supply in dog carcass samples (Canis lupus familiaris) by arterial injection with Indian ink and radiographic contrast. Int. J. Morphol., 36(2):407-412, 2018.

SUMMARY: The rupture of the cranial cruciate ligament is one of the most common causes of lameness in the dog, and etiology of this disease is not yet clear. The diagnosis of the pathology is performed by clinical orthopedic tests, typically the drawer test and the tibial compression test. The development of different surgical techniques and treatments incorporate surgical osteotomies at the tibial tuberosity level, but the procedure may not be successful due to post-surgical bone ischemia. The descriptions of the vascular anatomy of this joint area are scarce and remain poorly represented in anatomical texts. The objective of this study was to describe the origin of the blood supply to the proximal epiphysis and diaphysis at its proximal third of the tibia in the dog. Contrast medium mixed with Indian ink was injected into two different arterial pathways, visualized with simple radiography prior to the injection and contrasted radiography postinjection. A total of 48 pelvic limbs of dogs were evaluated and organized in three groups based upon the arterial injection pathway. The group injected through the popliteal artery, as well as the group injected through the femoral artery, was observed to have staining in the tibial tuberosity.

KEY WORDS: Anatomy; Dog; Blood supply; Tibia.

\section{REFERENCIAS BIBLIOGRÁFICAS}

AhChong, K.; Chiu, K. M.; Lo, S. F.; Iu, P. P. \& Yip, A. W. Arterial lesions in severe lower limb ischaemia: a prospective study of 100 consecutive ischaemic limbs in a Hong Kong Chinese population. Aust. N. Z. J. Surg., 69(1):48-51, 1999.

Álvarez, A. Tratamiento de la rotura del ligamento cruzado anterior en perros - Una visión global. Rev. Vet. Focus, 21(2):39-46, 2011.

Cook, J. L. Cranial cruciate ligament disease in dogs: biology versus biomechanics. Vet. Surg., 39(3):270-7, 2010.
Córdoba, M.; Montenegro, I. \& García, O. ¿Otra técnica nueva para resolver el mismo problema de siempre? Rev. Complut. Cienc. Vet., 1(2):316-22, 2007.

Dehghani, S.; Vafafar, A. \& Emami, M. J. Angiographic study of the canine tibial fracture fixed by bovine bone pins as compared with the Conventional metal pins. Iran. J. Vet. Res., 5(2):67-72, 2004.

Evans, H. E. \& Delahunta, A. Disección del Perro. $5^{\text {th }}$ ed. Ciudad de México, McGraw-Hill Interamericana, 2002.

Kato, F.; Basset, F. H. 3rd \& Silver, D. Arteriography of the nutrient artery and blood flow of the canine tibia. J. Bone Joint Surg. Am., 52(7):13766, 1970.

Kanamiya, T.; Naito, M.; Ikari, N. \& Hara, M. The effect of surgical dissections on blood flow to the tibial tubercle. J. Orthop. Res., 19(1):113-6, 2001.

König, H. E. \& Liebich, H. G. Anatomía de los Animales Domésticos, Aparato Locomotor. $2^{\text {nd }}$ ed. Buenos Aires, Médica Panamericana. 2005.

Mendoza, J. Conceptos Básicos de Radiología Veterinaria. Buenos Aires, Intermédica. 2001.

Parra, G. J.; Corvalán, Z. F.; Ibáñez, C. F., Bianchi, S. V.; Bastías, W.; Salas, C. C.; Cam, L. A. \& Lamarca, T. A. Atrapamiento de la arteria poplítea: Comunicación de un caso. Rev. Med. Chile, 134(2):207-10, 2006.

Rodríguez Quirós, J.; Guerrero, T.; San Román Ascaso, F. \& Jiménez Socorro, A. Rotura del ligamento cruzado en el perro: Signos clínicos, diagnóstico y tratamiento. Canis Felis, 95:6-26, 2008.

Steinberg, E. J.; Prata, R. G.; Palazzini, K. \& Brown, D. C. Tibial tuberosity advancement for treatment of CrCL injury: complications and owner satisfaction. J. Am. Anim. Hosp. Assoc., 47(4):250-7, 2011.

\author{
Dirección para correspondencia \\ Dr. Oliver Quintana García \\ Universidad Santo Tomás \\ Camino Catemito 1830 \\ San Bernardo \\ Santiago \\ CHILE
}

Email: oquintanag@santotomas.cl

Recibido : 14-12-2017

Aceptado: 30-01-2018 